\title{
On the Dimension of the Set of Two-View Multi-Homography Matrices
}

\author{
Wojciech Chojnacki and Anton van den Hengel
}

\begin{abstract}
It is shown that the set of all multi-homography matrices describing $I$-element families of interdependent homographies between two views has dimension $4 I+7$.
\end{abstract}

Mathematics Subject Classification (2010). Primary 14P10, 14Q99; Secondary 51N15.

Keywords. multi-homography matrix, semi-algebraic set, manifold, dimension.

\section{Introduction}

Let $\mathbb{R}$ denote the set of real numbers and let $\mathbb{R}^{m \times n}$ denote the set of $m \times n$ matrices with entries in $\mathbb{R}$. We identify coordinate vectors in $\mathbb{R}^{n}$ with $n \times 1$ matrices in $\mathbb{R}^{n \times 1}$, or, what is the same, with length- $n$ column vectors with real entries. Given $\mathbf{A} \in \mathbb{R}^{3 \times 3}, \mathbf{b} \in \mathbb{R}^{3},\left\{\mathbf{v}_{i}\right\}_{i=1}^{I} \subset \mathbb{R}^{3}$ and $\left\{w_{i}\right\}_{i=1}^{I} \subset \mathbb{R}$, let, for every $i=1, \ldots, I, \mathbf{H}_{i}$ be the $3 \times 3$ matrix defined by

$$
\mathbf{H}_{i}=w_{i} \mathbf{A}+\mathbf{b v}_{i}^{\top}
$$

where the superscript $\mathrm{T}$ denotes transposition. As it turns out (see below), each $\mathbf{H}_{i}$, provided that it is invertible, is a homography matrix for a homography of specific geometric significance, acting in two-dimensional real projective space. For each $i=1, \ldots, I$, let $\mathbf{h}_{i}=\operatorname{vec}\left(\mathbf{H}_{i}\right)$, where vec denotes column-wise vectorisation [9], and let $\mathbf{H}$ be the $9 \times I$ matrix given by

$$
\mathbf{H}=\left[\mathbf{h}_{1}, \ldots, \mathbf{h}_{I}\right] .
$$

Henceforth any $\mathbf{H}=\mathbf{H}\left(\mathbf{A}, \mathbf{b}, \mathbf{v}_{1}, \ldots, \mathbf{v}_{I}, w_{1}, \ldots, w_{I}\right)$ of this form, irrespective of whether the underlying constituent matrices $\mathbf{H}_{i}$ are invertible or not, will be referred to as a two-view multi-homography matrix, or simply as a multi-homography matrix. The set of all multi-homography matrices will be denoted by $\mathcal{H}$. The present paper addresses the problem of the computation of the dimension of $\mathcal{H}$. The notion of dimension that is of relevance here has to do with the fact $\mathcal{H}$ is a polynomial image of $\mathbb{R}^{4 I+12}$. Recall that a 
map $\mathbf{f}=\left[f_{1}, \ldots, f_{n}\right]^{\top}: \mathbb{R}^{m} \rightarrow \mathbb{R}^{n}$ is said to be polynomial if the functions $f_{i}=f_{i}(\mathbf{x})$ are polynomial functions in the entries of the vector argument $\mathbf{x}=\left[x_{1}, \ldots, x_{m}\right]^{\top}$. The celebrated Tarski-Seidenberg theorem $[1,2]$ ensures that the image of any polynomial map $\mathbf{f}: \mathbb{R}^{m} \rightarrow \mathbb{R}^{n}$ is a semi-algebraic set - that is, a finite union of sets, each defined by a finite conjunction of polynomial equalities and inequalities with real coefficients. Any semialgebraic set is locally a submanifold on a dense open subset. This permits defining the dimension of a semi-algebraic set to be the largest dimension at points around which the set is a submanifold.

The present paper reveals that the dimension of the semi-algebraic set $\mathcal{H}$ is equal to $4 I+7$. This result has its origins in computer vision in the context of solving certain statistical parameter estimation problems [3-5]. One issue that arises naturally in connection with these problems is the question of characterising the Zariski closure of $\mathcal{H}$, which is the smallest set containing $\mathcal{H}$ defined by finitely many polynomials with real coefficients, as a set of points satisfying explicit constraints put on the ambient Euclidean space. While some constraints - like the so-called rank-four constraint (to be discussed later) - have been identified, a full set of constraints has not been found yet. It is hoped that the dimensionality result established here will facilitate the task of uncovering a complete set of relevant constraints.

\section{Geometric link}

We start by explaining the geometric meaning of the matrices introduced in the Introduction.

Recall that if $V$ is a vector space, then the projective space $P(V)$ of $V$ is the set of one-dimensional vector subspaces of $V$. We write $P\left(\mathbb{R}^{n+1}\right)$ as $P^{n}(\mathbb{R})$. Any one-dimensional subspace of $P^{n}(\mathbb{R})$ is the set of all multiples of a non-zero vector in $\mathbb{R}^{n+1}$. Given $\mathbf{x}=\left[x_{1}, \ldots, x_{n+1}\right]^{\top} \in \mathbb{R}^{n+1} \backslash\{0\}$, let $[\mathbf{x}] \in P^{n}(\mathbb{R})$ be the set of all multiples of $\mathbf{x}$. Then $\mathbf{x}$ is said to be a representative vector for $[\mathbf{x}]$. If $\rho \neq 0$, then $\rho \mathbf{x}$ is another representative vector for $[\mathbf{x}]$ so that $[\mathbf{x}]=[\rho \mathbf{x}]$. Any member $\underline{\mathbf{x}}=\left[x_{1}, \ldots, x_{n}\right]^{\top}$ of $\mathbb{R}^{n}$ can be identified with the point $[\mathbf{x}]$ in $P^{n}(\mathbb{R})$ with $\mathbf{x}=\left[x_{1}, \ldots, x_{n}, 1\right]^{\top}$; the vector $\mathbf{x}$ is then called the homogeneous vector for $\underline{\mathbf{x}}$. The part of $P^{n}(\mathbb{R})$ identified with $\mathbb{R}^{n}$ consists of the so-called ordinary points of $P^{n}(\mathbb{R})$, the remaining part $P^{n}(\mathbb{R}) \backslash \mathbb{R}^{n}$ being comprised of the so-called ideal points of $P^{n}(\mathbb{R})$.

Given a linear map $\mathbf{A}$, let $\mathcal{R}(\mathbf{A})$ and $\mathcal{N}(\mathbf{A})$ denote the range space and the null space of $\mathbf{A}$, respectively. For a matrix $\mathbf{A}$, let $\mathcal{R}(\mathbf{A})$ and $\mathcal{N}(\mathbf{A})$ denote the column space (or the range) and the column null space (or the kernel) of A, respectively.

If $\mathbf{H}$ is an $(n+1) \times(n+1)$ invertible matrix, then $\mathbf{H}$ gives rise to a homography $P(\mathbf{H}): P^{n}(\mathbb{R}) \rightarrow P^{n}(\mathbb{R})$ given by

$$
P(\mathbf{H})([\mathbf{x}])=[\mathbf{H} \mathbf{x}], \quad \mathbf{x} \in \mathbb{R}^{n+1} .
$$

If $\rho \neq 0$, then $\rho \mathbf{H}$ and $\mathbf{H}$ define the same homography, and any matrix of the form $\rho \mathbf{H}$ is a homography matrix for $P(\mathbf{H})$. If $\mathbf{P}$ is an $(n+1) \times(m+1)$ 


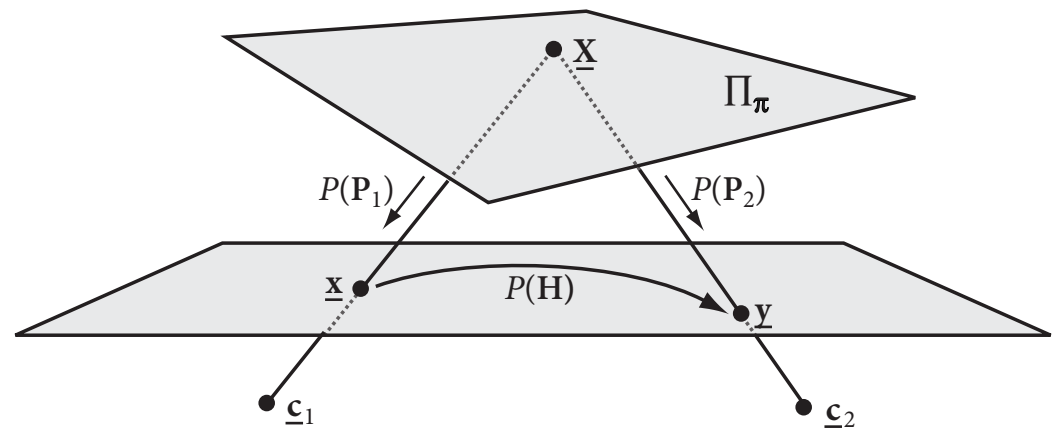

FiguRE 1. Homography between two views induced by a plane.

matrix with $n<m$ and of rank $n+1$, then $C=P(\mathcal{N}(\mathbf{P}))$ is a projective subspace of $P^{m}(\mathbb{R})$ of dimension $m-n-1$ and $\mathbf{P}$ gives rise to a projection $P(\mathbf{P}): P^{m}(\mathbb{R}) \backslash C \rightarrow P^{n}(\mathbb{R})$ from the centre $C$ given by

$$
P(\mathbf{P})([\mathbf{x}])=[\mathbf{P} \mathbf{x}], \quad \mathbf{x} \in \mathbb{R}^{m+1} .
$$

If $\rho \neq 0$, then $\rho \mathbf{P}$ and $\mathbf{P}$ define the same projection, and any matrix of the form $\rho \mathbf{P}$ is a projection matrix for $P(\mathbf{P})$.

Any non-zero vector $\pi \in \mathbb{R}^{n+1}$ defines the hyperplane in $P^{n}(\mathbb{R})$

$$
\Pi_{\boldsymbol{\pi}}=\left\{[\mathbf{x}] \in P^{n}(\mathbb{R}) \mid \boldsymbol{\pi}^{\top} \mathbf{x}=0\right\},
$$

with all non-zero multiples of $\boldsymbol{\pi}$ defining the same hyperplane.

Let $\mathbf{P}_{1}$ and $\mathbf{P}_{2}$ be two $3 \times 4$ matrices given by

$$
\mathbf{P}_{1}=\left[\mathbf{I}_{3}, \mathbf{0}\right] \text { and } \mathbf{P}_{2}=[\mathbf{A},-\mathbf{b}],
$$

where $\mathbf{I}_{3}$ is the $3 \times 3$ identity matrix, $\mathbf{0}$ is the length- 3 zero vector, and $\mathbf{A} \in$ $\mathbb{R}^{3 \times 3}$ and $\mathbf{b} \in \mathbb{R}^{3}$ are such that $\mathbf{P}_{2}$ has rank 3 . The matrices $\mathbf{P}_{1}$ and $\mathbf{P}_{2}$ give rise to two projections $P\left(\mathbf{P}_{1}\right): P^{3}(\mathbb{R}) \rightarrow P^{2}(\mathbb{R})$ and $P\left(\mathbf{P}_{2}\right): P^{3}(\mathbb{R}) \rightarrow P^{2}(\mathbb{R})$ with zero-dimensional (point) centres $C_{1} \in P^{3}(\mathbb{R})$ and $C_{2} \in P^{3}(\mathbb{R})$. The centre $C_{1}$ actually lies in $\mathbb{R}^{3}$ and is represented by the vector $\underline{\mathbf{c}}_{1}=[0,0,0]^{\top}$. Suppose that the other centre also lies in $\mathbb{R}^{3}$ and is represented by a length- 3 vector $\underline{\mathbf{c}}_{2}$. Let $\boldsymbol{\pi}=\left[\mathbf{v}^{\top}, w\right]^{\top}$ be a length- 4 vector with $\mathbf{v} \in \mathbb{R}^{3}$ and $w \in \mathbb{R}$, and let $\Pi_{\boldsymbol{\pi}}$ be the corresponding plane in $P^{3}(\mathbb{R})$. Then, associated with $P\left(\mathbf{P}_{1}\right)$, $P\left(\mathbf{P}_{2}\right)$, and $\Pi_{\boldsymbol{\pi}}$, there is a specific homography acting in $P^{2}(\mathbb{R})$. The action of this homography on the ordinary points of $P^{2}(\mathbb{R})$ can be described as follows. Given $\underline{\mathbf{x}} \in \mathbb{R}^{2} \subset P^{2}(\mathbb{R})$, issue a line through $\underline{\mathbf{c}}_{1}$ and $\underline{\mathbf{x}}$ and let $\underline{\mathbf{X}}$ be the point of intersection of this line and $\Pi_{\boldsymbol{\pi}}$. Next issue a line through $\underline{\mathbf{X}}$ and $\underline{\mathbf{c}}_{2}$ and let $\mathbf{y}$ be the point of intersection of this line and $\mathbb{R}^{2}$. The mapping that takes $\underline{\mathbf{x}}$ to $\mathbf{y}$ is the homography in question (see Figure 1). It can be shown that this homography can be represented as $P(\mathbf{H})$ with

$$
\mathbf{H}=w \mathbf{A}+\mathbf{b v}^{\top} ;
$$


in other words, if $\underline{\mathbf{x}}$ and $\underline{\mathbf{y}}$ are represented by respective homogeneous vectors $\mathbf{x}$ and $\mathbf{y}$, then

$$
[\mathbf{y}]=[\mathbf{H x}]
$$

(see [8]). The mapping $P(\mathbf{H})$ is termed the homography induced by the plane $\Pi_{\boldsymbol{\pi}}$ between the views described by $P\left(\mathbf{P}_{1}\right)$ and $P\left(\mathbf{P}_{2}\right)$.

With $\mathbf{P}_{1}$ and $\mathbf{P}_{2}$ as above, if $\left\{\boldsymbol{\pi}_{i}\right\}_{i=1}^{I}$ is a set of length- 4 vectors $\boldsymbol{\pi}_{i}=\left[\mathbf{v}_{i}^{\top}, w_{i}\right]^{\top}$ with $\mathbf{v}_{i} \in \mathbb{R}^{3}$ and $w_{i} \in \mathbb{R}$, then, for each $i=1, \ldots, I$, the $i$-th plane $\Pi_{\boldsymbol{\pi}_{i}}$ induces a homography $P\left(\mathbf{H}_{i}\right)$ with

$$
\mathbf{H}_{i}=w_{i} \mathbf{A}+\mathbf{b v}_{i}^{\top} .
$$

These homographies are all interlinked, as they are all generated under the common views described by $P\left(\mathbf{P}_{1}\right)$ and $P\left(\mathbf{P}_{2}\right)$.

\section{Algebro-geometric prerequisites}

Let $\mathbb{R}\left[x_{1}, \ldots, x_{n}\right]$ denote the set of all polynomials in the indeterminates $x_{1}, \ldots, x_{n}$ with real coefficients. A subset $V$ of $\mathbb{R}^{n}$ is a variety or an algebraic set if there exist polynomials $p_{1}, \ldots, p_{m}$ in $\mathbb{R}\left[x_{1}, \ldots, x_{n}\right]$ such that

$$
V=\mathbb{V}\left(p_{1}, \ldots, p_{m}\right),
$$

where

$$
\mathbb{V}\left(p_{1}, \ldots, p_{m}\right)=\left\{\mathbf{x} \in \mathbb{R}^{n} \mid p_{\mu}(\mathbf{x})=0 \quad \text { for all } 1 \leq \mu \leq m\right\} .
$$

A subset $S$ of $\mathbb{R}^{n}$ is a semi-algebraic set if

$$
S=\bigcup_{\mu=1}^{m} \bigcap_{\nu=1}^{n_{\mu}}\left\{\mathbf{x} \in \mathbb{R}^{n} \mid p_{\mu, \nu}(\mathbf{x}) \triangleright_{\mu \nu} 0\right\},
$$

where $p_{\mu, \nu}$ are polynomials in $\mathbb{R}\left[x_{1}, \ldots, x_{n}\right]$ and $\triangleright_{\mu \nu}$ is one of the three relational operators $<,=,>$. In other words, a semi-algebraic set is a finite union of sets, each determined by a finite number of polynomial equations and inequalities with real coefficients.

A map $\mathbf{f}: S \rightarrow T$, where $S \subset \mathbb{R}^{n}$ and $T \subset \mathbb{R}^{m}$ are semi-algebraic sets, is semi-algebraic if the graph of $\mathbf{f}$,

$$
\left\{\left[\mathbf{x}^{\top}, \mathbf{f}(\mathbf{x})^{\top}\right]^{\top} \in \mathbb{R}^{n+m} \mid \mathbf{x} \in S\right\},
$$

is a semi-algebraic subset of $\mathbb{R}^{n+m}$. If $\mathbf{f}=\left[f_{1}, \ldots, f_{m}\right]^{\top}$ is a polynomial map, then $\mathbf{f}$ is semi-algebraic because its graph can be described by $m$ polynomial equalities

$$
y_{\mu}-f_{\mu}(\mathbf{x})=0 \quad(1 \leq \mu \leq m) .
$$

A key result about semi-algebraic sets is the Tarski-Seidenberg theorem saying that if $S \subset \mathbb{R}^{n}$ and $T \subset \mathbb{R}^{m}$ are semi-algebraic sets and $\mathbf{f}: S \rightarrow T$ is a semi-algebraic map, then the image $\mathbf{f}(S) \subset T$ is a semi-algebraic set [1,2]. In particular, the images of polynomial maps are semi-algebraic. 


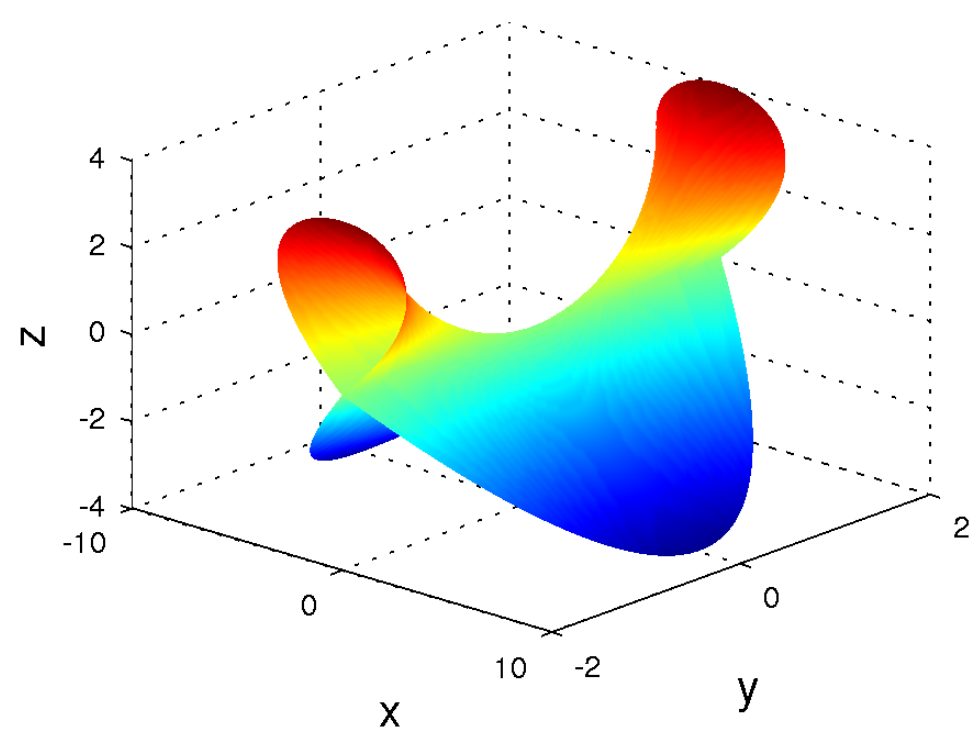

Figure 2. Plot of a portion of the variety $\mathbb{V}\left(x^{2}-y^{2} z^{2}+z^{3}\right)$.

Some semi-algebraic sets are smooth manifolds and some are not. Consider, for example, the image in $\mathbb{R}^{3}$ of $\mathbb{R}^{2}$ by the polynomial map

$$
(t, u) \mapsto\left(t\left(u^{2}-t^{2}\right), u, u^{2}-t^{2}\right) .
$$

It coincides with the variety $\mathbb{V}\left(x^{2}-y^{2} z^{2}+z^{3}\right)$. This variety is not a smooth manifold because, locally, at each point of the $y$-axis other than the origin, the surface looks like the intersection of two smooth manifolds - see Figure 2.

While not all semi-algebraic sets are manifolds, it turns out that every semi-algebraic set can be meaningfully assigned a dimension. This is a consequence of the fact that every semi-algebraic set admits a stratification. To get an idea of the concept, consider again the variety $\mathbb{V}\left(x^{2}-y^{2} z^{2}+z^{3}\right)$. This variety can be represented as the set-theoretic union of several twodimensional surfaces together with a one-dimensional smooth manifold, the $y$-axis. These smooth manifolds constitute a stratification of $\mathbb{V}\left(x^{2}-y^{2} z^{2}+z^{3}\right)$.

Formally, a stratification of a set $X \subset \mathbb{R}^{n}$ is a finite partition $\left\{X_{i}\right\}_{i \in I}$ of $X$ such that

(S1) each $X_{i}$, called a stratum of $X$, is a $d_{i}$-dimensional smooth manifold in $\mathbb{R}^{n}$;

(S2) (frontier condition) if $X_{j} \cap \overline{X_{i}} \neq \emptyset$, then $X_{j} \subset \overline{X_{i}}$ and $d_{j}<d_{i}{ }^{1}$ where $\bar{Y}$ denotes the closure of $Y$.

A stratification is called semi-algebraic if every stratum is semi-algebraic. A stratified set is a set that admits a stratification. The dimension of a stratified

\footnotetext{
${ }^{1}$ As the strata are disjoint, this means that either $X_{i}=X_{j}$ or $X_{i} \subset \overline{X_{j}} \backslash X_{j}$.
} 
set is the largest dimension of a stratum. A fundamental result about semialgebraic sets is that every such set has a semi-algebraic stratification $[1,2]$.

\section{Main result}

Our set of interest $\mathcal{H}$ is a polynomial image of $\mathbb{R}^{4 I+12}$ (see Section 5.1). Consequently, $\mathcal{H}$ is semi-algebraic and one can speak about its dimension. The main result which we shall establish is the following:

Theorem. The dimension of $\mathcal{H}$ is equal to $4 I+7$.

We shall split the proof of this theorem into two parts, corresponding to the two inequalities: $\operatorname{dim} \mathcal{H} \leq 4 I+7$ and $\operatorname{dim} \mathcal{H} \geq 4 I+7$. The first inequality has already surfaced in the literature [5], but the derivation of it that we present here is in some aspects new. The second inequality is novel and constitutes the main contribution of the paper.

\section{Upper dimension bound}

We first show that $\operatorname{dim} \mathcal{H} \leq 4 I+7$. With a view to providing some perspective on our main result, we start by presenting a number of weaker bounds on the dimension of $\mathcal{H}$ obtained earlier and only then do we derive the ultimate bound $\operatorname{dim} \mathcal{H} \leq 4 I+7$.

\subsection{Initial upper bounds}

Let $\mathbf{H}$ be a multi-homography matrix associated with $\mathbf{A} \in \mathbb{R}^{3 \times 3}, \mathbf{b} \in \mathbb{R}^{3}$, $\left\{\mathbf{v}_{i}\right\}_{i=1}^{I} \subset \mathbb{R}^{3}$ and $\left\{w_{i}\right\}_{i=1}^{I} \subset \mathbb{R}$. Then, with $\mathbf{a}=\operatorname{vec}(\mathbf{A})$, for each $i=1, \ldots, I$, the $i$ th column $\mathbf{h}_{i}$ of $\mathbf{H}$ can be written as

$$
\mathbf{h}_{i}=w_{i} \operatorname{vec}(\mathbf{A})+\operatorname{vec}\left(\mathbf{b v}_{i}^{\top}\right)=w_{i} \mathbf{a}+\left(\mathbf{I}_{3} \otimes \mathbf{b}\right) \mathbf{v}_{i},
$$

where $\otimes$ denotes Kronecker product [9]. This implies that

$$
\mathbf{H}=\mathbf{S T},
$$

where $\mathbf{S}$ is the $9 \times 4$ matrix given by

$$
\mathbf{S}=\left[\mathbf{I}_{3} \otimes \mathbf{b}, \mathbf{a}\right]
$$

and $\mathbf{T}$ is the $4 \times I$ matrix given by

$$
\mathbf{T}=\left[\begin{array}{ccc}
\mathbf{v}_{1} & \ldots & \mathbf{v}_{I} \\
w_{1} & \ldots & w_{I}
\end{array}\right] .
$$

An immediate consequence of (5.2) is that, whenever $I \geq 4, \mathbf{H}$ has rank at most 4 . In other words,

$$
\mathcal{H} \subset \mathbb{R}_{4}^{9 \times I} \text { for } I \geq 4,
$$

this being the rank-four constraint mentioned in the Introduction [10] (see also [12]). Here $\mathbb{R}_{k}^{m \times n}$ denotes the set of real $m \times n$ matrices of rank at most $k$. It is well known that $\mathbb{R}_{k}^{m \times n}$ is a $k(m+n-k)$-dimensional variety in $\mathbb{R}^{m \times n}[7]$. 
In particular, $\operatorname{dim} \mathbb{R}_{4}^{9 \times I}=4(9+I-4)=4 I+20$ for $I \geq 4$. Combining this with (5.3) yields $\operatorname{dim} \mathcal{H} \leq 4 I+20$ for $I \geq 4$.

A stronger bound can be obtained by noting explicitly that any multihomography matrix $\mathbf{H}$ can be naturally expressed in terms of an underlying array of parameters

$$
\boldsymbol{\omega}=\left(\mathbf{A}, \mathbf{b}, \mathbf{v}_{1}, \ldots, \mathbf{v}_{I}, w_{1}, \ldots, w_{I}\right),
$$

where $\mathbf{A} \in \mathbb{R}^{3 \times 3}, \mathbf{b} \in \mathbb{R}^{3},\left\{\mathbf{v}_{i}\right\}_{i=1}^{I} \subset \mathbb{R}^{3}$ and $\left\{w_{i}\right\}_{i=1}^{I} \subset \mathbb{R}$. More specifically, if $\boldsymbol{\Pi}(\boldsymbol{\omega})$ is the $3 \times 3 I$ matrix given by

$$
\boldsymbol{\Pi}(\boldsymbol{\omega})=\left[\boldsymbol{\Pi}_{1}(\boldsymbol{\omega}), \ldots, \boldsymbol{\Pi}_{I}(\boldsymbol{\omega})\right],
$$

where

$$
\boldsymbol{\Pi}_{i}(\boldsymbol{\omega})=w_{i} \mathbf{A}+\mathbf{b v}_{i}^{\top}
$$

for each $i=1, \ldots, I$, then

$$
\mathbf{H}=r(\boldsymbol{\Pi}(\boldsymbol{\omega})),
$$

where $r$ denotes the reshaping map

$$
\left[\mathbf{M}_{1}, \ldots, \mathbf{M}_{I}\right] \mapsto\left[\operatorname{vec}\left(\mathbf{M}_{1}\right), \ldots, \operatorname{vec}\left(\mathbf{M}_{I}\right)\right]
$$

with $\mathbf{M}_{i} \in \mathbb{R}^{3 \times 3}$ for each $i=1, \ldots, I$. While the array $\boldsymbol{\omega}$ has entries of different types, it can always be reshaped to a length- $(4 I+12)$ vector, for example

$$
\left[\operatorname{vec}(\mathbf{A})^{\top}, \mathbf{b}^{\top}, \mathbf{v}_{1}^{\top}, \ldots, \mathbf{v}_{I}^{\top}, w_{1}, \ldots, w_{I}\right]^{\top},
$$

and be viewed as an element of $\mathbb{R}^{4 I+12}$. Consequently, the set $\Omega$ of all arrays $\boldsymbol{\omega}$ as above has dimension $4 I+12$. As (5.5) says that $\mathcal{H}$ is the image of $\Omega$ under the composite mapping $r \circ \boldsymbol{\Pi}$ and as $r \circ \boldsymbol{\Pi}$ is smooth, we conclude that $\operatorname{dim} \mathcal{H} \leq 4 I+12$.

This estimate can be further refined to the inequality $\operatorname{dim} \mathcal{H} \leq 4 I+$ 10 [3]. Indeed, it follows from (5.1) that any multi-homography matrix $\mathbf{H}$ splits as the sum

$$
\mathbf{H}=\mathbf{H}^{\prime}+\mathbf{H}^{\prime \prime},
$$

where

$$
\mathbf{H}^{\prime}=\left[w_{1} \mathbf{a}, \ldots, w_{I} \mathbf{a}\right]=\mathbf{a w}^{T}, \quad \mathbf{w}=\left[w_{1}, \ldots, w_{I}\right]^{\top}
$$

and

$$
\mathbf{H}^{\prime \prime}=\left[\left(\mathbf{I}_{3} \otimes \mathbf{b}\right) \mathbf{v}_{1}, \ldots\left(\mathbf{I}_{3} \otimes \mathbf{b}\right) \mathbf{v}_{I}\right]=\left(\mathbf{I}_{3} \otimes \mathbf{b}\right) \mathbf{V}, \quad \mathbf{V}=\left[\mathbf{v}_{1}, \ldots, \mathbf{v}_{I}\right] .
$$

Clearly, $\mathbf{H}^{\prime}$ is a rank-one $9 \times I$ matrix. Corresponding to $\mathbf{H}^{\prime \prime}$, define a $3 \times 3 I$ matrix $\mathbf{H}_{0}^{\prime \prime}$ by

$$
\mathbf{H}_{0}^{\prime \prime}=\left[\mathbf{b} \mathbf{v}_{1}^{\top}, \ldots, \mathbf{b} \mathbf{v}_{I}^{\top}\right]=\mathbf{b}\left[\mathbf{v}_{1}^{\top}, \ldots, \mathbf{v}_{I}^{\top}\right] .
$$

The factorisation in the rightmost term shows that $\mathbf{H}_{0}^{\prime \prime}$ has rank one. Now, $\mathbf{H}^{\prime \prime}=r\left(\mathbf{H}_{0}^{\prime \prime}\right)$, and so

$$
\mathbf{H}=\mathbf{H}^{\prime}+r\left(\mathbf{H}_{0}^{\prime \prime}\right) \text {. }
$$

Given that the varieties $\mathbb{R}_{1}^{9 \times I}$ and $\mathbb{R}_{1}^{3 \times 3 I}$ to which $\mathbf{H}^{\prime}$ and $\mathbf{H}_{0}^{\prime \prime}$ belong have dimensions $I+8$ and $3 I+2$, respectively, and that $r$ is smooth, we find that

$$
\operatorname{dim} \mathcal{H} \leq(I+8)+(3 I+2)=4 I+10 .
$$




\subsection{Ultimate upper bound}

A still better, in fact optimal, upper estimate of the dimension of $\mathcal{H}$ is $\operatorname{dim} \mathcal{H} \leq 4 I+7[5]$. We shall derive it by exploiting the fact there are many different parameter arrays describing one and the same multi-homography matrix. Our derivation will pursue a slightly different path than that taken in $[5]$.

For each matrix

$$
\mathbf{C}=\left[\begin{array}{llll}
\alpha & 0 & 0 & c_{1} \\
0 & \alpha & 0 & c_{2} \\
0 & 0 & \alpha & c_{3} \\
0 & 0 & 0 & \beta
\end{array}\right]
$$

where $\alpha, \beta \in \mathbb{R} \backslash\{0\}$ and $\mathbf{c}=\left[c_{1}, c_{2}, c_{3}\right]^{\top} \in \mathbb{R}^{3}$, let $\tau_{\mathbf{C}}$ be the transformation of $\Omega$ into itself given by

$$
\begin{aligned}
\tau_{\mathbf{C}}(\boldsymbol{\omega})= & \left(\beta \mathbf{A}+\mathbf{b c}^{\top}, \alpha \mathbf{b}\right. \\
& \alpha^{-1} \mathbf{v}_{1}-\alpha^{-1} \beta^{-1} \mathbf{c}, \ldots, \alpha^{-1} \mathbf{v}_{I}-\alpha^{-1} \beta^{-1} \mathbf{c}, \\
& \left.\beta^{-1} w_{1}, \ldots, \beta^{-1} w_{I}\right)
\end{aligned}
$$

With the matrix composition as group operation and with the $4 \times 4$ identity matrix $\mathbf{I}_{4}$ as neutral element, the set $G$ of all matrices $\mathbf{C}$ as above is a group. Denote by $\operatorname{Aut}(\Omega)$ the set of all one-to-one transformations of $\Omega$. Under the composition of mappings as group operation and with the identity mapping of $\Omega$ as neutral element, $\operatorname{Aut}(\Omega)$ is a group. It is readily verified that the function $\tau: \mathbf{C} \mapsto \tau_{\mathbf{C}}$ maps $G$ into $\operatorname{Aut}(\Omega)$ (so that each $\tau_{\mathbf{C}}$ is a bijection) and is a homomorphism:

$$
\tau_{\mathbf{C}} \tau_{\mathbf{C}^{\prime}}=\tau_{\mathbf{C C}^{\prime}}, \quad \tau_{\mathbf{C}}^{-1}=\tau_{\mathbf{C}^{-1}}
$$

for any $\mathbf{C}, \mathbf{C}^{\prime} \in G$. A critical property of the $\tau_{\mathbf{C}}$ 's is that each of these transformations leaves all the homography matrices unchanged:

$$
\Pi\left(\tau_{\mathbf{C}}(\boldsymbol{\omega})\right)=\boldsymbol{\Pi}(\boldsymbol{\omega})
$$

for every $\boldsymbol{\omega} \in \Omega$. Thus the $\tau_{\mathbf{C}}$ 's constitute a group of internal symmetries related to the freedom of choice of parameter arrays. The fact that $\tau$ is a homomorphism can be phrased as saying that $\tau$ is a representation of $G$ in the gauge group. The latter group comprises all transformations $\gamma$ in $\operatorname{Aut}(\Omega)$ such that $\boldsymbol{\Pi}(\gamma(\boldsymbol{\omega}))=\boldsymbol{\Pi}(\boldsymbol{\omega})$ for each $\boldsymbol{\omega} \in \Omega$. Under the equivalence relation in which $\boldsymbol{\omega}, \boldsymbol{\omega}^{\prime} \in \Omega$ are regarded as equivalent whenever $\boldsymbol{\omega}^{\prime}=\tau_{\mathbf{C}}(\boldsymbol{\omega})$ for some $\mathbf{C} \in G$, the set $\Omega$ is partitioned into classes of intrinsically equivalent parameter arrays, with each class representing exactly one underlying multihomography matrix. While these classes can vary in size with changing $\boldsymbol{\omega}$, the majority of them - and this is a crucial observation - can be identified with $G$ and hence have dimension 5 . We elaborate on this point and its consequences next. 
Let

$$
\begin{aligned}
& \Omega_{1}=\{\boldsymbol{\omega} \in \Omega \mid \mathbf{b}=\mathbf{0}\}, \\
& \Omega_{2}=\left\{\boldsymbol{\omega} \in \Omega \mid \mathbf{b} \neq \mathbf{0}, w_{i}=0 \text { for each } i=1, \ldots, I\right\}, \\
& \Omega_{3}=\Omega \backslash\left(\Omega_{1} \cup \Omega_{2}\right) .
\end{aligned}
$$

Note that each of the above three sets is $\tau_{\mathbf{C}}$-invariant for every $\mathbf{C} \in G$. It is clear that $\boldsymbol{\Pi}\left(\Omega_{1}\right)$ consists of the matrices of the form $\left[w_{1} \mathbf{A}, \ldots, w_{I} \mathbf{A}\right]$, whereas $\boldsymbol{\Pi}\left(\Omega_{2}\right)$ consists of the matrices of the form $\left[\mathbf{b v}_{1}^{\top}, \ldots, \mathbf{b} \mathbf{v}_{I}^{\top}\right]$. Taking into account that the inverse mapping $r^{-1}$ is smooth ( $r$ is clearly one-to-one) and $\left[w_{1} \mathbf{A}, \ldots, w_{I} \mathbf{A}\right]=r^{-1}\left(\mathbf{a w}^{\top}\right)$, and reusing the argument from the last paragraph of the previous subsection, we conclude that $\operatorname{dim} \Pi\left(\Omega_{1}\right) \leq I+8$ and $\operatorname{dim} \Pi\left(\Omega_{2}\right) \leq 3 I+2$. We shall prove shortly that $\operatorname{dim} \Pi\left(\Omega_{3}\right) \leq 4 I+7$. Assuming this for now, note that together the last three inequalities imply that

$$
\operatorname{dim} \Pi(\Omega) \leq 4 I+7
$$

At this point, observe that $\Pi(\Omega)$ coincides with $r^{-1}(\mathcal{H})$ - see (5.5). Note, moreover, that as $r$ is a one-to-one smooth mapping, $r$ and $r^{-1}$ do not change the dimensions of sets that they transform. Consequently,

$$
\operatorname{dim} \mathcal{H}=\operatorname{dim} r^{-1}(\mathcal{H})=\operatorname{dim} \Pi(\Omega)
$$

Combining this with (5.7) yields the desired bound $\operatorname{dim} \mathcal{H} \leq 4 I+7$.

To prove that $\operatorname{dim} \Pi\left(\Omega_{3}\right) \leq 4 I+7$, it suffices to show that, for each $\boldsymbol{\omega} \in \Omega_{3}$, the class of $\boldsymbol{\omega}$ under the action of the $\tau_{\mathbf{C}}$ 's can be identified with $G$. Indeed, if this is established, then

$$
\begin{aligned}
\operatorname{dim} \Pi\left(\Omega_{3}\right) & \leq \operatorname{dim} \Omega_{3}-\operatorname{dim} G \leq \operatorname{dim} \Omega-\operatorname{dim} G \\
& =(4 I+12)-5=4 I+7 .
\end{aligned}
$$

We shall show that the mapping $\mathbf{C} \mapsto \tau_{\mathbf{C}}(\boldsymbol{\omega})$ is one-to-one for each $\boldsymbol{\omega} \in \Omega_{3}$. It suffices to prove that $\tau_{\mathbf{C}}(\boldsymbol{\omega})=\boldsymbol{\omega}$ implies $\mathbf{C}=\mathbf{I}_{4}$ for each $\boldsymbol{\omega} \in \Omega_{3}$. Take an arbitrary $\boldsymbol{\omega} \in \Omega_{3}$. Then $\mathbf{b} \neq \mathbf{0}$ and $w_{i_{0}} \neq 0$ for some $i_{0} \in\{1, \ldots, I\}$. If $\tau_{\mathbf{C}}(\boldsymbol{\omega})=\boldsymbol{\omega}$ holds for some $\mathbf{C}$ as given in (5.6), then $\beta^{-1} w_{i_{0}}=w_{i_{0}}, \alpha \mathbf{b}=\mathbf{b}$, and $\alpha^{-1} \mathbf{v}_{1}-\alpha^{-1} \beta^{-1} \mathbf{c}=\mathbf{v}_{1}$. The first of these equalities implies that $\beta=1$, the second implies that $\alpha=1$, and the third together with $\alpha=\beta=1$ implies that $\mathbf{c}=\mathbf{0}$. Thus $\mathbf{C}=\mathbf{I}_{4}$, as desired.

\section{Lower dimension bound}

Here we show that $\operatorname{dim} \mathcal{H} \geq 4 I+7$. This together with the last result of the previous section will imply that $\operatorname{dim} \mathcal{H}=4 I+7$ and will finish the proof of our theorem. 


\subsection{Initial reduction}

Let $\Omega_{0}$ be the set of those $\boldsymbol{\omega}$ in $\Omega$ for which

$$
\|\mathbf{b}\|^{2}=\mathbf{b}^{\top} \mathbf{b}=1 \text {. }
$$

As pointed out earlier, $\Omega$ is essentially identical with the Euclidean space $\mathbb{R}^{4 I+12}$. Accordingly, $\Omega_{0}$ can be viewed as a hypersurface in $\mathbb{R}^{4 I+12}$. Consider the restriction $\left.\Pi\right|_{\Omega_{0}}$ of the map $\Pi$ to $\Omega_{0}$,

$$
\left.\boldsymbol{\Pi}\right|_{\Omega_{0}}: \Omega_{0} \rightarrow \mathbb{R}^{3 \times 3 I},\left.\quad \boldsymbol{\Pi}\right|_{\Omega_{0}}(\boldsymbol{\omega})=\Pi(\boldsymbol{\omega}), \quad \boldsymbol{\omega} \in \Omega_{0} .
$$

Note that the image of $\Omega_{0}$ by $\left.\boldsymbol{\Pi}\right|_{\Omega_{0}}$,

$$
\left.\Pi\right|_{\Omega_{0}}\left(\Omega_{0}\right)=\boldsymbol{\Pi}\left(\Omega_{0}\right),
$$

is equal to the image $\boldsymbol{\Pi}(\Omega)$ of $\Omega$ by $\boldsymbol{\Pi}$. Indeed, given $\boldsymbol{\omega} \in \Omega$, the right-hand side of (5.4) does not change if $\boldsymbol{\omega}$ is replaced by $\boldsymbol{\omega}_{0} \in \Omega_{0}$ defined as the modification of $\boldsymbol{\omega}$ in which (i) if $\mathbf{b} \neq \mathbf{0}$, then $\|\mathbf{b}\|^{-1} \mathbf{b}$ is substituted for $\mathbf{b}$ and, for each $i=1, \ldots, I,\|\mathbf{b}\| \mathbf{v}_{i}$ is substituted for $\mathbf{v}_{i}$, and (ii) if $\mathbf{b}=\mathbf{0}$, then an arbitrary length-3 vector $\mathbf{b}_{0}$ with $\left\|\mathbf{b}_{0}\right\|=1$ is substituted for $\mathbf{b}$ and all the $\mathbf{v}_{i}$ 's are taken to be zero, with the rest of the entries of $\boldsymbol{\omega}$ remaining unaltered in either case. Now, in view of (5.8), to complete the argument, it suffices to show that $\operatorname{dim} \Pi\left(\Omega_{0}\right) \geq 4 I+7$.

Given $\boldsymbol{\omega} \in \Omega$, denote by $\mathrm{d} \boldsymbol{\Pi}_{\boldsymbol{\omega}}$ the differential (or the linearisation) of $\boldsymbol{\Pi}$ at $\boldsymbol{\omega}$. For $\boldsymbol{\omega} \in \Omega_{0}$, denote by $T_{\boldsymbol{\omega}}\left(\Omega_{0}\right)$ the tangent space of $\Omega_{0}$ at $\boldsymbol{\omega}$ and by $\mathrm{d}\left(\left.\boldsymbol{\Pi}\right|_{\Omega_{0}}\right)_{\boldsymbol{\omega}}$ the differential of $\left.\boldsymbol{\Pi}\right|_{\Omega_{0}}$ at $\boldsymbol{\omega}$. When a particular local parametrisation $\sigma$ for $\Omega_{0}$ is chosen together with $\mathbf{p} \in \mathbb{R}^{4 I+11}$ satisfying $\sigma(\mathbf{p})=\boldsymbol{\omega}, \mathrm{d}\left(\left.\boldsymbol{\Pi}\right|_{\Omega_{0}}\right)_{\boldsymbol{\omega}}$ can be identified with the Jacobian matrix of the composite mapping $\boldsymbol{\Pi} \circ \sigma$ at p. As it turns out, the dimension of $\boldsymbol{\Pi}\left(\Omega_{0}\right)$ is identical with the rank of $\mathrm{d}\left(\left.\boldsymbol{\Pi}\right|_{\Omega_{0}}\right)_{\boldsymbol{\omega}}$ calculated at any $\boldsymbol{\omega}$ belonging to some generic subset of $\boldsymbol{\Pi}\left(\Omega_{0}\right)$. We shall explain this rather delicate point in the next subsection.

\subsection{Regular points}

First we recall a few concepts from differential topology, including those of a regular point and a regular value of a smooth mapping. Because our mapping of interest $\left.\boldsymbol{\Pi}\right|_{\Omega_{0}}$ is not locally injective or surjective, we shall use a slightly generalised definition of regular point and regular value.

Given a linear map $\mathbf{A}$, denote by $\operatorname{rank} \mathbf{A}$ and null $\mathbf{A}$ the rank and the nullity of $\mathbf{A}$; that is,

$$
\operatorname{rank} \mathbf{A}=\operatorname{dim} \mathcal{R}(\mathbf{A}) \quad \text { and } \quad \text { null } \mathbf{A}=\operatorname{dim} \mathcal{N}(\mathbf{A}) .
$$

Let $f: X \rightarrow Y$ be a smooth map between smooth manifolds $X$ and $Y$. Let $\mathrm{r}_{\max }(f)$ be the maximal rank of $\mathrm{d} f_{\mathbf{x}}$ for any $\mathbf{x} \in X$. A point $\mathbf{x} \in X$ is called a regular point of $f$ if $\mathrm{d} f_{\mathbf{x}}$ has $\operatorname{rank} \mathrm{r}_{\max }(f)$, and is called a critical point of $f$ if $\mathrm{d} f_{\mathbf{x}}$ has rank less than $\mathrm{r}_{\max }(f)$. A point $\mathbf{y} \in Y$ is a regular value of $f$ if every $\mathbf{x} \in f^{-1}(\{\mathbf{y}\})$ is a regular point; this includes the case where $f^{-1}(\{\mathbf{y}\})$ is empty. Otherwise, $\mathbf{y}$ is called a critical value of $f$. We denote by $\operatorname{Reg}(f)$ the set of regular points of $f$, and by $\operatorname{Crit}(f)$ the set of critical points 
of $f$. With this notation, the set of critical values of $f$ is nothing else but $f(\operatorname{Crit}(f))$, and the set of regular values $f$ coincides with $Y \backslash f(\operatorname{Crit}(f))$.

The principal result of this subsection is the following equality:

$$
\mathrm{r}_{\max }\left(\left.\boldsymbol{\Pi}\right|_{\Omega_{0}}\right)=\operatorname{dim} \boldsymbol{\Pi}\left(\Omega_{0}\right) .
$$

It reduces the calculation of $\operatorname{dim} \boldsymbol{\Pi}\left(\Omega_{0}\right)$ to the calculation of $\mathrm{r}_{\max }\left(\left.\boldsymbol{\Pi}\right|_{\Omega_{0}}\right)$.

We start by showing that $\mathrm{r}_{\max }\left(\left.\boldsymbol{\Pi}\right|_{\Omega_{0}}\right) \leq \operatorname{dim} \boldsymbol{\Pi}\left(\Omega_{0}\right)$. As is known, if $\operatorname{rankd}\left(\left.\boldsymbol{\Pi}\right|_{\Omega_{0}}\right)_{\boldsymbol{\omega}_{0}}=\mathrm{r}_{\max }\left(\left.\boldsymbol{\Pi}\right|_{\Omega_{0}}\right)$ for some $\boldsymbol{\omega}_{0} \in \Omega_{0}$, then $\operatorname{rank} \mathrm{d}\left(\left.\boldsymbol{\Pi}\right|_{\Omega_{0}}\right)_{\boldsymbol{\omega}}=$ $\mathrm{r}_{\max }\left(\left.\boldsymbol{\Pi}\right|_{\Omega_{0}}\right)$ for all $\boldsymbol{\omega}$ in some open neighbourhood of $\boldsymbol{\omega}_{0}$ in $\Omega_{0}[11, \S 11.2]$. In particular, if rank $\mathrm{d}\left(\left.\boldsymbol{\Pi}\right|_{\Omega_{0}}\right)_{\boldsymbol{\omega}}=\mathrm{r}_{\max }\left(\left.\boldsymbol{\Pi}\right|_{\Omega_{0}}\right)$ for some $\boldsymbol{\omega}_{0} \in \Omega_{0}$, then $\mathrm{d}\left(\left.\boldsymbol{\Pi}\right|_{\Omega_{0}}\right)_{\boldsymbol{\omega}}$ has constant rank $\mathrm{r}_{\max }\left(\left.\boldsymbol{\Pi}\right|_{\Omega_{0}}\right)$ for all $\boldsymbol{\omega}$ in a open neighbourhood of $\boldsymbol{\omega}_{0}$. This property combined with the constant rank theorem [11, Thm. 11.1] guarantees that if $\boldsymbol{\omega} \in \Omega_{0}$ is such that $\operatorname{rankd}\left(\left.\boldsymbol{\Pi}\right|_{\Omega_{0}}\right)_{\boldsymbol{\omega}}=\mathrm{r}_{\max }\left(\left.\boldsymbol{\Pi}\right|_{\Omega_{0}}\right)$, then there is an open neighbourhood $U \subset \Omega_{0}$ of $\boldsymbol{\omega}$ such that $\boldsymbol{\Pi}(U)$ is a $\mathrm{r}_{\max }\left(\left.\boldsymbol{\Pi}\right|_{\Omega_{0}}\right)$ dimensional regular (embedded) submanifold of $\mathbb{R}^{4 I+12}$. It follows that $\boldsymbol{\Pi}\left(\Omega_{0}\right)$ contains a $\mathrm{r}_{\max }\left(\left.\boldsymbol{\Pi}\right|_{\Omega_{0}}\right)$-dimensional submanifold, and hence $\mathrm{r}_{\max }\left(\left.\boldsymbol{\Pi}\right|_{\Omega_{0}}\right) \leq$ $\operatorname{dim} \Pi\left(\Omega_{0}\right)$.

We now prove that $\mathrm{r}_{\max }\left(\left.\boldsymbol{\Pi}\right|_{\Omega_{0}}\right) \geq \operatorname{dim} \boldsymbol{\Pi}\left(\Omega_{0}\right)$. Let $\left\{S_{i}\right\}_{i \in I}$ be a (finite) semi-algebraic stratification of $\Pi\left(\Omega_{0}\right)$, with $d_{i}$ the dimension of $S_{i}$ for each $i \in I$. Let $S_{i_{0}}$ be any stratum of $\boldsymbol{\Pi}\left(\Omega_{0}\right)$ of maximum dimension, i.e.,

$$
\operatorname{dim} S_{i_{0}}=\operatorname{dim} \Pi\left(\Omega_{0}\right) .
$$

Let

$$
X=\left.\Pi\right|_{\Omega_{0}} ^{-1}\left(S_{i_{0}}\right)
$$

We claim that $X$ is an open subset of $\Omega_{0}$.

To establish the claim, we first show that for each $\mathbf{M} \in S_{i_{0}}$ there is an open set $U_{\mathbf{M}} \subset \mathbb{R}^{3 \times 3 I}$ containing $\mathbf{M}$ such that

$$
U_{\mathbf{M}} \cap \mathbf{\Pi}\left(\Omega_{0}\right)=U_{\mathbf{M}} \cap S_{i_{0}} .
$$

Assume the contrary. Then there exists $\mathbf{M} \in S_{i_{0}}$ such that for every open set $U \subset \mathbb{R}^{3 \times 3 I}$ containing $\mathbf{M}$, there is $i \neq i_{0}$ such that $U_{\mathbf{M}} \cap S_{i} \neq \emptyset$. Consequently, there exists a sequence $\left\{\mathbf{M}_{n}\right\}_{n=1}^{\infty}$ of matrices in $\mathbb{R}^{3 \times 3 I}$ such that $\lim _{n \rightarrow \infty} \mathbf{M}_{n}=\mathbf{M}$ and, for each positive integer $n, \mathbf{M}_{n}$ is in $S_{i_{n}}$ with $i_{n} \neq i_{0}$. Since the index set $I$ is finite, we can extract a subsequence $\left\{\mathbf{M}_{n_{k}}\right\}_{k=1}^{\infty}$ from $\left\{\mathbf{M}_{n}\right\}_{n=1}^{\infty}$ such that all the $\mathbf{M}_{n_{k}}$ 's belong to one and the same stratum $S_{j}$ different from $S_{i_{0}}$. Then, clearly, $\mathbf{M}$ is in $\overline{S_{j}}$, and we see that the set $S_{i_{0}} \cap \overline{S_{j}}$, containing $\mathbf{M}$, is non-empty. By the frontier condition (S2), $S_{i_{0}} \subset S_{j}$ and $d_{i_{0}}<d_{j}$. But this contradicts $d_{i_{0}}$ being the maximum of all the $d_{i}$ 's.

Having established the existence of $U_{\mathbf{M}}$ satisfying (6.3) for each $\mathbf{M} \in$ $S_{i_{0}}$, we now note that, by the continuity of $\left.\boldsymbol{\Pi}\right|_{\Omega_{0}},\left.\boldsymbol{\Pi}\right|_{\Omega_{0}} ^{-1}\left(U_{\mathbf{M}}\right)$ is an open subset of $\Omega_{0}$ for each $\mathbf{M} \in S_{i_{0}}$. Since

$$
\left.\boldsymbol{\Pi}\right|_{\Omega_{0}} ^{-1}\left(U_{\mathbf{M}}\right)=\left.\boldsymbol{\Pi}\right|_{\Omega_{0}} ^{-1}\left(U_{\mathbf{M}} \cap \mathbf{\Pi}\left(\Omega_{0}\right)\right)
$$

and, in view of $(6.3)$,

$$
\left.\boldsymbol{\Pi}\right|_{\Omega_{0}} ^{-1}\left(U_{\mathbf{M}} \cap \mathbf{\Pi}\left(\Omega_{0}\right)\right)=\left.\mathbf{\Pi}\right|_{\Omega_{0}} ^{-1}\left(U_{\mathbf{M}} \cap S_{i_{0}}\right),
$$


it follows that $\left.\boldsymbol{\Pi}\right|_{\Omega_{0}} ^{-1}\left(U_{\mathbf{M}} \cap S_{i_{0}}\right)$ is an open subset of $\Omega_{0}$ for each $\mathbf{M} \in S_{i_{0}}$. But

$$
X=\left.\bigcup_{\mathbf{M} \in S_{i_{0}}} \boldsymbol{\Pi}\right|_{\Omega_{0}} ^{-1}\left(U_{\mathbf{M}} \cap S_{i_{0}}\right),
$$

and this together with the preceding statement implies that $X$ is an open subset of $\Omega_{0}$, as claimed.

In particular, $X$ is a smooth manifold in its own right and the restriction $\left.\boldsymbol{\Pi}\right|_{X}$ of $\boldsymbol{\Pi}$ to $X$ is a smooth map from $X$ to $\mathbb{R}^{3 \times 3 I}$. Since $S_{i_{0}}$ is a regular (embedded) submanifold of $\mathbb{R}^{3 \times 3 I},\left.\boldsymbol{\Pi}\right|_{X}$ induces a smooth map $\tilde{\boldsymbol{\Pi}}_{X}: X \rightarrow S_{i_{0}}$ between manifolds [11, Thm. 11.20]. If $i$ denotes the natural embedding of $S_{i_{0}}$ into $\mathbb{R}^{3 \times 3 I}$, then $\tilde{\boldsymbol{\Pi}}_{X}$ and $\left.\boldsymbol{\Pi}\right|_{X}$ are linked by the relation

$$
\left.\boldsymbol{\Pi}\right|_{X}=i \circ \tilde{\boldsymbol{\Pi}}_{X}
$$

Since, by construction, $\left.\boldsymbol{\Pi}\right|_{X}$ maps $X$ onto $S_{i_{0}}$, it follows that also $\tilde{\boldsymbol{\Pi}}_{X}$ maps $X$ onto $S_{i_{0}}$. By the classical theorem of Sard [6, Chap. 1, $\S 1$ ], the set $\tilde{\boldsymbol{\Pi}}_{X}\left(\operatorname{Crit}\left(\tilde{\boldsymbol{\Pi}}_{X}\right)\right)$ of critical values of $\tilde{\boldsymbol{\Pi}}_{X}$ has $\left(\operatorname{dim} S_{i_{0}}\right)$-dimensional measure zero, and, because $\tilde{\boldsymbol{\Pi}}_{X}$ is surjective, we have

$$
\mathrm{r}_{\max }\left(\tilde{\boldsymbol{\Pi}}_{X}\right)=\operatorname{dim} S_{i_{0}} .
$$

In particular, $\operatorname{Reg}\left(\tilde{\boldsymbol{\Pi}}_{X}\right)$ is non-empty and

$$
\operatorname{rankd}\left(\tilde{\boldsymbol{\Pi}}_{X}\right)_{\boldsymbol{\omega}}=\operatorname{dim} S_{i_{0}}
$$

for each $\boldsymbol{\omega} \in \operatorname{Reg}\left(\tilde{\boldsymbol{\Pi}}_{X}\right)$. In view of (6.4),

$$
\left.\mathrm{d} \Pi\right|_{X}=\mathrm{d} i \cdot \mathrm{d} \tilde{\Pi}_{X}
$$

by the chain rule, and, as $\mathrm{d} i$ is injective, we have

$$
\operatorname{rankd}\left(\tilde{\boldsymbol{\Pi}}_{X}\right)_{\boldsymbol{\omega}}=\operatorname{rankd}\left(\left.\boldsymbol{\Pi}\right|_{X}\right)_{\boldsymbol{\omega}}
$$

for every $\boldsymbol{\omega} \in X$, so that

$$
\mathrm{r}_{\max }\left(\left.\boldsymbol{\Pi}\right|_{X}\right)=\mathrm{r}_{\max }\left(\tilde{\boldsymbol{\Pi}}_{X}\right) .
$$

This equality together with (6.5) implies

$$
\mathrm{r}_{\max }\left(\left.\boldsymbol{\Pi}\right|_{X}\right)=\operatorname{dim} S_{i_{0}} .
$$

Since, obviously, $\mathrm{r}_{\max }\left(\left.\boldsymbol{\Pi}\right|_{X}\right) \leq \mathrm{r}_{\max }(\boldsymbol{\Pi})$, it follows that

$$
\operatorname{dim} \boldsymbol{\Pi}\left(\Omega_{0}\right)=\operatorname{dim} S_{i_{0}} \leq \mathrm{r}_{\max }(\boldsymbol{\Pi}),
$$

as was to be shown.

\subsection{Generic points}

By virtue of (6.2), all we need is to estimate from below the rank of $\mathrm{d}\left(\left.\boldsymbol{\Pi}\right|_{\Omega_{0}}\right)_{\boldsymbol{\omega}}$ at some $\boldsymbol{\omega} \in \operatorname{Reg}\left(\left.\boldsymbol{\Pi}\right|_{\Omega_{0}}\right)$. In order to proceed with the actual estimation, we shall first have to be able to exclude points at which our calculations might break down. As it turns out, a systematic procedure for excluding such exceptional points can be devised based on the fact that $\operatorname{Reg}\left(\left.\boldsymbol{\Pi}\right|_{\Omega_{0}}\right)$ is a so-called Zariski open subset of $\Omega_{0}$. 
Let $\boldsymbol{R}: \Omega \rightarrow \mathbb{R}^{3 \times 3 I} \times \mathbb{R}$ be the mapping defined by

$$
\boldsymbol{R}(\boldsymbol{\omega})=[\boldsymbol{\Pi}(\boldsymbol{\omega}), f(\boldsymbol{\omega})], \quad f(\boldsymbol{\omega})=\|b\|^{2}-1, \quad \boldsymbol{\omega} \in \Omega .
$$

Note that, given $\boldsymbol{\omega} \in \Omega_{0}$, a vector $\delta \boldsymbol{\omega} \in T_{\boldsymbol{\omega}}(\Omega)$ lies in the subspace $T_{\boldsymbol{\omega}}\left(\Omega_{0}\right) \subset T_{\boldsymbol{\omega}}(\Omega)$ if and only if $\mathrm{d} f_{\boldsymbol{\omega}}(\delta \boldsymbol{\omega})=0$. This observation together with the equality

$$
\mathrm{d} \boldsymbol{R}_{\boldsymbol{\omega}}(\delta \boldsymbol{\omega})=\left[\mathrm{d} \boldsymbol{\Pi}_{\boldsymbol{\omega}}(\delta \boldsymbol{\omega}), \mathrm{d} f_{\boldsymbol{\omega}}(\delta \boldsymbol{\omega})\right]
$$

implies that

$$
\mathcal{N}\left(\mathrm{d} \boldsymbol{R}_{\boldsymbol{\omega}}\right)=\mathcal{N}\left(\left.\mathrm{d} \boldsymbol{\Pi}_{\boldsymbol{\omega}}\right|_{T_{\boldsymbol{\omega}}\left(\Omega_{0}\right)}\right)
$$

for any $\boldsymbol{\omega} \in \Omega_{0}$. As $\left.\mathrm{d} \boldsymbol{\Pi}_{\boldsymbol{\omega}}\right|_{T_{\boldsymbol{\omega}}\left(\Omega_{0}\right)}=\mathrm{d}\left(\left.\boldsymbol{\Pi}\right|_{\Omega_{0}}\right)_{\boldsymbol{\omega}}$ for $\boldsymbol{\omega} \in \Omega_{0}$, we see that

$$
\mathcal{N}\left(\mathrm{d} \boldsymbol{R}_{\boldsymbol{\omega}}\right)=\mathcal{N}\left(\mathrm{d}\left(\left.\boldsymbol{\Pi}\right|_{\Omega_{0}}\right)_{\boldsymbol{\omega}}\right)
$$

and further that

$$
\operatorname{null} \mathrm{d} \boldsymbol{R}_{\boldsymbol{\omega}}=\operatorname{null} \mathrm{d}\left(\left.\boldsymbol{\Pi}\right|_{\Omega_{0}}\right)_{\boldsymbol{\omega}}
$$

for any $\boldsymbol{\omega} \in \Omega_{0}$. We also have

$$
\operatorname{rank} \mathrm{d}\left(\left.\boldsymbol{\Pi}\right|_{\Omega_{0}}\right)_{\boldsymbol{\omega}}+\operatorname{null} \mathrm{d}\left(\left.\boldsymbol{\Pi}\right|_{\Omega_{0}}\right)_{\boldsymbol{\omega}}=\operatorname{dim} T_{\boldsymbol{\omega}}\left(\Omega_{0}\right)
$$

for any $\boldsymbol{\omega} \in \Omega_{0}$. At the level of the Jacobian matrices, this is nothing else but an instance of the rank-nullity law of linear algebra saying that the rank and the nullity of a matrix add up to the number of columns of the matrix. Now, by definition, a member $\boldsymbol{\omega}$ of $\Omega_{0}$ is in $\operatorname{Crit}\left(\left.\boldsymbol{\Pi}\right|_{\Omega_{0}}\right)$ if and only if

$$
\operatorname{rankd}\left(\left.\boldsymbol{\Pi}\right|_{\Omega_{0}}\right)_{\boldsymbol{\omega}}<\mathrm{r}_{\max }\left(\left.\boldsymbol{\Pi}\right|_{\Omega_{0}}\right) \text {. }
$$

Equivalently, in view of (6.7), $\boldsymbol{\omega} \in \Omega_{0}$ is in $\operatorname{Crit}\left(\left.\boldsymbol{\Pi}\right|_{\Omega_{0}}\right)$ if and only if

$$
\operatorname{null} \mathrm{d}\left(\left.\boldsymbol{\Pi}\right|_{\Omega_{0}}\right)_{\boldsymbol{\omega}}>\operatorname{dim} T_{\boldsymbol{\omega}}\left(\Omega_{0}\right)-\mathrm{r}_{\max }\left(\left.\boldsymbol{\Pi}\right|_{\Omega_{0}}\right) \text {. }
$$

Note that, in analogy to (6.7), we have

$$
\text { null } \mathrm{d} \boldsymbol{R}_{\boldsymbol{\omega}}+\operatorname{rank} \mathrm{d} \boldsymbol{R}_{\boldsymbol{\omega}}=\operatorname{dim} T_{\boldsymbol{\omega}}(\Omega)=\operatorname{dim} T_{\boldsymbol{\omega}}\left(\Omega_{0}\right)+1
$$

for every $\boldsymbol{\omega} \in \Omega$. This in conjunction with (6.6) and (6.8) implies that $\boldsymbol{\omega} \in \Omega_{0}$ is in $\operatorname{Crit}\left(\left.\boldsymbol{\Pi}\right|_{\Omega_{0}}\right)$ if and only if

$$
\operatorname{rank} \mathrm{d} \boldsymbol{R}_{\boldsymbol{\omega}}<\mathrm{r}_{\max }\left(\left.\boldsymbol{\Pi}\right|_{\Omega_{0}}\right)+1 .
$$

Choosing standard Cartesian coordinates for $\Omega$ and representing each $\mathrm{d} \boldsymbol{R}_{\boldsymbol{\omega}}$ by a corresponding Jacobi matrix, we see that (6.9) holds if and only if all the $\left(\mathrm{r}_{\max }\left(\left.\boldsymbol{\Pi}\right|_{\Omega_{0}}\right)+1\right) \times\left(\mathrm{r}_{\max }\left(\left.\boldsymbol{\Pi}\right|_{\Omega_{0}}\right)+1\right)$ minors of $\mathrm{d} \boldsymbol{R}_{\boldsymbol{\omega}}$ vanish. Therefore the set $V$ of all $\boldsymbol{\omega} \in \Omega$ satisfying (6.9) is algebraic. Moreover, $\Omega_{0}$ is algebraic as well-in fact, $\Omega_{0}$ is the product algebraic set $\mathbb{R}^{4 I+9} \times \mathbb{S}^{2}$, where $\mathbb{S}^{2}$ denotes the two-dimensional unit sphere in $\mathbb{R}^{3}$. Since $\operatorname{Crit}\left(\left.\boldsymbol{\Pi}\right|_{\Omega_{0}}\right)$ is the intersection of $V$ with $\Omega_{0}$, it follows that $\operatorname{Crit}\left(\left.\boldsymbol{\Pi}\right|_{\Omega_{0}}\right)$ is a subvariety of $\Omega_{0}$ - that is, a set obtained from $\Omega_{0}$ by imposing additional polynomial equations.

Recall that a variety is called irreducible if it cannot be represented as a union of two proper subvarieties. It is a basic fact that a variety $V \subset \mathbb{R}^{n}$ is irreducible if and only if the following property holds: if the product of two polynomials in $\mathbb{R}\left[x_{1}, \ldots, x_{n}\right]$ vanishes identically on $V$, then one of the polynomials vanishes identically on $V$; in other words, the set of all polynomials in $\mathbb{R}\left[x_{1}, \ldots, x_{n}\right]$ vanishing identically on $V$ is a prime ideal 
of the ring $\mathbb{R}\left[x_{1}, \ldots, x_{n}\right]$. Since the product of two irreducible varieties is irreducible and since both $\mathbb{R}^{4 I+9}$ and $\mathbb{S}^{2}$ are irreducible (the irreducibility of $\mathbb{R}^{n}$ for any positive integer $n$ is a standard result which stems from the fact that $\mathbb{R}\left[x_{1}, \ldots, x_{n}\right]$ is an integral domain, and for the irreducibility of $\mathbb{S}^{2}$ see Appendix A), it follows that $\Omega_{0}$ is an irreducible variety. Thus $\operatorname{Crit}\left(\left.\boldsymbol{\Pi}\right|_{\Omega_{0}}\right)$ is a proper subvariety of the irreducible variety $\Omega_{0}$.

In algebraic geometry, a subvariety of a variety $V$ is alternatively called a Zariski closed subset of $V$. As it turns out, a union of a finite number of a proper Zariski closed subsets of an irreducible variety is always a proper subset. Accordingly, a proper subvariety of an irreducible variety may be considered a "small" subset. A complement of a Zariski closed subset of a variety $V$ is termed a Zariski open subset of $V$. Zariski open subsets of an irreducible variety are "large" - the intersection of any finite number of nonempty Zariski open subsets of an irreducible variety is always non-empty. Using the above terminology, $\operatorname{Crit}\left(\left.\boldsymbol{\Pi}\right|_{\Omega_{0}}\right)$ is a Zariski closed subset of $\Omega_{0}$ and as such is "small", and $\operatorname{Reg}\left(\left.\boldsymbol{\Pi}\right|_{\Omega_{0}}\right)$ is a Zariski open subset of $\Omega_{0}$ and hence is "large".

The benefit of identifying $\operatorname{Reg}\left(\left.\boldsymbol{\Pi}\right|_{\Omega_{0}}\right)$ as a Zariski open subset of $\Omega_{0}$ is that one can impose finitely many additional polynomial inequalities of the form $p(\boldsymbol{\omega}) \neq 0$, where $p$ does not vanish identically on $\Omega_{0},{ }^{2}$ to hold on $\operatorname{Reg}\left(\left.\boldsymbol{\Pi}\right|_{\Omega_{0}}\right)$ and still obtain a non-empty set. This is so because each inequality $p(\boldsymbol{\omega}) \neq 0$ defines an open Zariski subset of $\Omega_{0}$, and the final set on which all inequalities hold is the intersection of a finite number of non-empty Zariski open subsets of $\Omega_{0}$ - a non-empty set.

It is customary to say that a property holds generically on an irreducible algebraic set $V$, if it holds on a non-empty Zariski-open subset of $V$. We shall use this terminology in relation to $\Omega_{0}$. More specifically, we shall speak about a generic point of $\Omega_{0}$ as a member of some initially unspecified non-empty Zariski open subset of $\Omega_{0}$ which is intersected with, or - equivalently - is a subset of, $\operatorname{Reg}\left(\left.\boldsymbol{\Pi}\right|_{\Omega_{0}}\right)$. The subset can be made precise a posteriori as the aggregate all of whose elements of $\operatorname{Reg}\left(\left.\boldsymbol{\Pi}\right|_{\Omega_{0}}\right)$ that satisfy all the conditions imposed in the proof.

\subsection{Upper nullity bound}

Let $\boldsymbol{\omega}$ be a generic point in $\Omega_{0}$. First note that the dimension of $T_{\boldsymbol{\omega}}\left(\Omega_{0}\right)$ equals the dimension of $\Omega_{0}$ and this, in view of the constraint (6.1), equals $4 I+11$, one less than the dimension of $\Omega$. This together with (6.7) gives

$$
\operatorname{rankd}\left(\left.\boldsymbol{\Pi}\right|_{\Omega_{0}}\right)_{\boldsymbol{\omega}}=4 I+11-\operatorname{null} \mathrm{d}\left(\left.\boldsymbol{\Pi}\right|_{\Omega_{0}}\right)_{\boldsymbol{\omega}} .
$$

Remembering that $\left.\mathrm{d} \boldsymbol{\Pi}_{\boldsymbol{\omega}}\right|_{T_{\boldsymbol{\omega}}\left(\Omega_{0}\right)}=\mathrm{d}\left(\left.\boldsymbol{\Pi}\right|_{\Omega_{0}}\right)_{\boldsymbol{\omega}}$, it is clear that to establish that $\operatorname{dim} \boldsymbol{\Pi}\left(\Omega_{0}\right) \geq 4 I+7$ we need only show that null $\left.\mathrm{d} \boldsymbol{\Pi}_{\boldsymbol{\omega}}\right|_{T_{\boldsymbol{\omega}}\left(\Omega_{0}\right)} \leq 4$. Let

$$
\delta \boldsymbol{\omega}=\left(\delta \mathbf{A}, \delta \mathbf{b}, \delta \mathbf{v}_{1}, \ldots, \delta \mathbf{v}_{I}, \delta w_{1}, \ldots, \delta w_{I}\right)
$$

\footnotetext{
${ }^{2}$ By the Real Nullstellensatz [1,2], a polynomial $p(\boldsymbol{\omega})$ vanishes identically on $\Omega_{0}$ if and only if there exist finitely many polynomials $q_{1}(\boldsymbol{\omega}), \ldots, q_{n}(\boldsymbol{\omega})$ and a positive integer $m$ such that the polynomial $p^{2 m}(\boldsymbol{\omega})+q_{1}^{2}(\boldsymbol{\omega})+\ldots q_{n}^{2}(\boldsymbol{\omega})$ is divisible by $\|\mathbf{b}\|^{2}-1$.
} 
be a tangent vector to $\Omega_{0}$ at $\boldsymbol{\omega}$. In view of (6.1),

$$
\mathbf{b}^{\top} \delta \mathbf{b}=0 \text {. }
$$

For $\delta \boldsymbol{\omega}$ to fall into the null space of $\mathrm{d} \boldsymbol{\Pi}_{\boldsymbol{\omega}}$, it is necessary and sufficient that

$$
\mathrm{d}\left(\boldsymbol{\Pi}_{i}\right)_{\boldsymbol{\omega}}(\delta \boldsymbol{\omega})=\delta w_{i} \mathbf{A}+w_{i} \delta \mathbf{A}+\delta \mathbf{b v}_{i}^{\top}+\mathbf{b} \delta \mathbf{v}_{i}^{\top}=\mathbf{0}
$$

for each $i=1, \ldots, I$. Assume that $\delta \boldsymbol{\omega}$ is in $\mathcal{N}\left(\mathrm{d} \boldsymbol{\Pi}_{\boldsymbol{\omega}}\right)$ so that (6.11) holds. Pre-multiplying (6.11) by $\mathbf{b}^{\top}$ and using (6.1) and (6.10) yields

$$
\delta w_{i} \mathbf{b}^{\top} \mathbf{A}+w_{i} \mathbf{b}^{\top} \delta \mathbf{A}+\delta \mathbf{v}_{i}^{\top}=\mathbf{0} .
$$

Pre-multiplying in turn this equation by $\mathbf{b}$ and subtracting the resulting equation from (6.11) leads to

$$
\delta w_{i}\left(\mathbf{I}_{3}-\mathbf{b b}^{\top}\right) \mathbf{A}+w_{i}\left(\mathbf{I}_{3}-\mathbf{b b}^{\top}\right) \delta \mathbf{A}+\delta \mathbf{b v}_{i}^{\top}=\mathbf{0} .
$$

The latter formula can be rewritten as

$$
\left(\mathbf{I}_{3}-\mathbf{b b}^{\top}\right)\left(\delta w_{i} \mathbf{A}+w_{i} \delta \mathbf{A}\right)+\delta \mathbf{b v}_{i}^{\top}=\mathbf{0},
$$

which upon post-multiplying by $\mathbf{v}_{i}$ gives

$$
\left(\mathbf{I}_{3}-\mathbf{b b}^{\top}\right)\left(\delta w_{i} \mathbf{A}+w_{i} \delta \mathbf{A}\right) \mathbf{v}_{i}+\delta \mathbf{b}\left\|\mathbf{v}_{i}\right\|^{2}=\mathbf{0} .
$$

Hence

$$
\delta \mathbf{b}=-\left(\mathbf{I}_{3}-\mathbf{b b}^{\top}\right)\left(\delta w_{i} \mathbf{A}+w_{i} \delta \mathbf{A}\right)\left\|\mathbf{v}_{i}\right\|^{-2} \mathbf{v}_{i} .
$$

Plugging this expression for $\delta \mathbf{b}$ back into (6.13), we find that

$$
\left(\mathbf{I}_{3}-\mathbf{b b}^{\top}\right)\left(\delta w_{i} \mathbf{A}+w_{i} \delta \mathbf{A}\right)\left(\mathbf{I}_{3}-\left\|\mathbf{v}_{i}\right\|^{-2} \mathbf{v}_{i} \mathbf{v}_{i}^{\top}\right)=\mathbf{0} .
$$

By virtue of the genericity of $\boldsymbol{\omega}$, we may assume that $w_{i} \neq 0$ for each $i=1, \ldots, I$, and the above equation can be restated as

$$
\left(\mathbf{I}_{3}-\mathbf{b b}^{\top}\right)\left(\frac{\delta w_{i}}{w_{i}} \mathbf{A}-\delta \mathbf{A}\right) \mathbf{P}_{\mathbf{v}_{i}}^{\perp}=\mathbf{0},
$$

where

$$
\mathbf{P}_{\mathbf{v}_{i}}^{\perp}=\mathbf{I}_{3}-\left\|\mathbf{v}_{i}\right\|^{-2} \mathbf{v}_{i} \mathbf{v}_{i}^{\top} .
$$

Another application of the genericity of $\boldsymbol{\omega}$ ensures that, given a pair $i$ and $j$ of distinct indices, the vectors $\mathbf{v}_{i}$ and $\mathbf{v}_{j}$ may be treated as linearly independent with their cross product $\mathbf{v}_{i} \times \mathbf{v}_{j}$ non-zero. Since

$$
\mathbf{v}_{i}^{\top}\left(\mathbf{v}_{i} \times \mathbf{v}_{j}\right)=\mathbf{v}_{j}^{\top}\left(\mathbf{v}_{i} \times \mathbf{v}_{j}\right)=\mathbf{0}
$$

we have

$$
\mathbf{P}_{\mathbf{v}_{i}}^{\perp}\left(\mathbf{v}_{i} \times \mathbf{v}_{j}\right)=\mathbf{P}_{\mathbf{v}_{j}}^{\perp}\left(\mathbf{v}_{i} \times \mathbf{v}_{j}\right)=\mathbf{v}_{i} \times \mathbf{v}_{j} .
$$

In view of (6.15),

$$
\left(\mathbf{I}_{3}-\mathbf{b b}^{\top}\right)\left(\frac{\delta w_{i}}{w_{i}} \mathbf{A}-\delta \mathbf{A}\right)\left(\mathbf{v}_{i} \times \mathbf{v}_{j}\right)=\mathbf{0}
$$

and

$$
\left(\mathbf{I}_{3}-\mathbf{b b}^{\top}\right)\left(\frac{\delta w_{j}}{w_{j}} \mathbf{A}-\delta \mathbf{A}\right)\left(\mathbf{v}_{i} \times \mathbf{v}_{j}\right)=\mathbf{0}
$$


Subtracting the second of these equations from the first, we obtain

$$
\left(\frac{\delta w_{i}}{w_{i}}-\frac{\delta w_{j}}{w_{j}}\right)\left(\mathbf{I}_{3}-\mathbf{b b}^{\top}\right) \mathbf{A}\left(\mathbf{v}_{i} \times \mathbf{v}_{j}\right)=\mathbf{0} .
$$

As, again by the genericity of $\boldsymbol{\omega}$, the vector $\left(\mathbf{I}_{3}-\mathbf{b b}^{\top}\right) \mathbf{A}\left(\mathbf{v}_{i} \times \mathbf{v}_{j}\right)$ may be assumed non-zero, we conclude that

$$
\frac{\delta w_{i}}{w_{i}}=\frac{\delta w_{j}}{w_{j}} .
$$

In other words, the $\delta w_{i} / w_{i}$ 's have a common value. Denote this value by $\delta \lambda$. Then (6.15) can be rewritten as

$$
\left(\mathbf{I}_{3}-\mathbf{b b}^{\top}\right)(\delta \lambda \mathbf{A}-\delta \mathbf{A}) \mathbf{P}_{\mathbf{v}_{i}}^{\perp}=\mathbf{0} .
$$

We now show that in fact

$$
\left(\mathbf{I}_{3}-\mathbf{b b}^{\top}\right)(\delta \lambda \mathbf{A}-\delta \mathbf{A})=\mathbf{0} .
$$

It suffices to prove that

$$
\left(\mathbf{I}_{3}-\mathbf{b b}^{\top}\right)(\delta \lambda \mathbf{A}-\delta \mathbf{A}) \mathbf{x}=\mathbf{0}
$$

for each length-3 vector $\mathbf{x}$. Choose two linearly independent vectors from amongst the $\mathbf{v}_{i}$ 's, say, $\mathbf{v}_{1}$ and $\mathbf{v}_{2}$. As any length-3 vector is a linear combination of $\mathbf{v}_{1}, \mathbf{v}_{2}$, and $\mathbf{v}_{1} \times \mathbf{v}_{2},(6.18)$ will be established once it is shown that it holds for $\mathbf{x}$ equal to $\mathbf{v}_{1}, \mathbf{v}_{2}$, and $\mathbf{v}_{1} \times \mathbf{v}_{2}$. Since $\mathbf{P}_{\mathbf{v}_{1}}^{\perp}\left(\mathbf{v}_{1} \times \mathbf{v}_{2}\right)=\mathbf{v}_{1} \times \mathbf{v}_{2}$, it follows from (6.16) that

$$
\begin{aligned}
\left(\mathbf{I}_{3}-\mathbf{b b}^{\top}\right)(\delta \lambda \mathbf{A}-\delta \mathbf{A})\left(\mathbf{v}_{1} \times \mathbf{v}_{2}\right) & \\
& =\left(\mathbf{I}_{3}-\mathbf{b b}^{\top}\right)(\delta \lambda \mathbf{A}-\delta \mathbf{A}) \mathbf{P}_{\mathbf{v}_{1}}^{\perp}\left(\mathbf{v}_{1} \times \mathbf{v}_{2}\right)=\mathbf{0},
\end{aligned}
$$

so (6.18) holds in the case $\mathbf{x}=\mathbf{v}_{1} \times \mathbf{v}_{2}$. Now

$$
\mathbf{v}_{1}=\left(1-\frac{\left(\mathbf{v}_{1}^{\top} \mathbf{v}_{2}\right)^{2}}{\left\|\mathbf{v}_{1}\right\|^{2}\left\|\mathbf{v}_{2}\right\|^{2}}\right)^{-1}\left(\frac{\mathbf{v}_{2}^{\top} \mathbf{v}_{1}}{\left\|\mathbf{v}_{2}\right\|^{2}} \mathbf{P}_{\mathbf{v}_{1}}^{\perp} \mathbf{v}_{2}+\mathbf{P}_{\mathbf{v}_{2}}^{\perp} \mathbf{v}_{1}\right)
$$

as direct verification shows. Using this representation together with (6.16) yields immediately

$$
\left(\mathbf{I}_{3}-\mathbf{b b}^{\top}\right)(\delta \lambda \mathbf{A}-\delta \mathbf{A}) \mathbf{v}_{1}=\mathbf{0} .
$$

Interchanging the roles of $\mathbf{v}_{1}$ and $\mathbf{v}_{2}$ in the above argument leads to

$$
\left(\mathbf{I}_{3}-\mathbf{b b}^{\top}\right)(\delta \lambda \mathbf{A}-\delta \mathbf{A}) \mathbf{v}_{2}=\mathbf{0} .
$$

Thus (6.18) also holds in the cases $\mathbf{x}=\mathbf{v}_{1}$ and $\mathbf{x}=\mathbf{v}_{2}$.

As an immediate consequence of (6.17), we obtain

$$
\begin{aligned}
\delta \mathbf{A} & =\mathbf{b b}^{\top} \delta \mathbf{A}+\left(\mathbf{I}_{3}-\mathbf{b b}^{\top}\right) \delta \mathbf{A} \\
& =\mathbf{b b}^{\top} \delta \mathbf{A}+\delta \lambda\left(\mathbf{I}_{3}-\mathbf{b}^{\top}\right) \mathbf{A} .
\end{aligned}
$$

Let $\delta \mathbf{c}$ be the length-3 vector defined by $\delta \mathbf{c}=\delta \mathbf{A b}$. Then

$$
\delta \mathbf{A}=\mathbf{b}(\delta \mathbf{c})^{\top}+\delta \lambda\left(\mathbf{I}_{3}-\mathbf{b b}^{\top}\right) \mathbf{A}
$$


expressing $\delta \mathbf{A}$ linearly in terms of $\delta \mathbf{c}$ and $\delta \lambda$. The relation

$$
\delta w_{i}=w_{i} \delta \lambda
$$

expresses $\delta w_{i}$ linearly in terms of $\delta \lambda$. Now (6.14) in which $\delta \mathbf{A}$ and $\delta w_{i}$ are replaced by the right-hand sides of (6.19) and (6.20), respectively, gives an expression for $\delta \mathbf{b}$ that is linear in $\delta \mathbf{c}$ and $\delta \lambda$. Finally, (6.12) rewritten as

$$
\delta \mathbf{v}_{i}=-\delta w_{i} \mathbf{A}^{\top} \mathbf{b}-w_{i}(\delta \mathbf{A})^{\top} \mathbf{b}
$$

and combined with (6.19) and (6.20) as in the previous step gives an expression for $\delta \mathbf{v}_{i}$ that is linear in $\delta \mathbf{c}$ and $\delta \lambda$. Thus all components of $\delta \boldsymbol{\omega}$ depend linearly on $\delta \mathbf{c}$ and $\delta \lambda$, which shows that the null space of $\left.\mathrm{d} \boldsymbol{\Pi}_{\boldsymbol{\omega}}\right|_{T_{\boldsymbol{\omega}}\left(\Omega_{0}\right)}$ is at most four dimensional. This completes the proof of the inequality $\operatorname{dim} \Pi\left(\Omega_{0}\right) \geq 4 I+7$.

\section{Acknowledgement}

This research was supported by the Australian Research Council.

\section{Appendix A. Irreducibility of the unit sphere}

Here we show that, for each positive integer $n$, the $n$-dimensional unit sphere

$$
\mathbb{S}^{n}=\left\{\mathbf{x} \in \mathbb{R}^{n+1} \mid x_{1}^{2}+\cdots+x_{n+1}^{2}=1\right\}
$$

is an irreducible real algebraic variety.

Given a positive integer $n$, suppose that $p_{1}$ and $p_{2}$ are two polynomials in $\mathbb{R}\left[x_{1}, \ldots, x_{n+1}\right]$ such that $p_{1}(\mathbf{x}) p_{2}(\mathbf{x})=0$ for each $\mathbf{x} \in \mathbb{S}^{n}$. We have to show that either $p_{1}(\mathbf{x})=0$ for each $\mathbf{x} \in \mathbb{S}^{n}$ or $p_{2}(\mathbf{x})=0$ for each $\mathbf{x} \in \mathbb{S}^{n}$. To this end, we parametrise $\mathbb{S}^{n}$ less the south pole $[0, \ldots, 0,1]^{\top} \in \mathbb{R}^{n+1}$ by $\mathbb{R}^{n}$ using the inverse of the stereographic projection from $[0, \ldots, 0,1]^{\top}$. Namely, we assign to each $\mathbf{u}=\left[u_{1}, \ldots, u_{n}\right]^{\top}$ the point $\left(\neq[0, \ldots, 0,1]^{\top}\right)$ where the line through $[0, \ldots, 0,1]^{\top}$ and $\left[u_{1}, \ldots, u_{n}, 0\right]^{\top}$ intersects $\mathbb{S}^{n}$. The algebraic formula capturing this geometric recipe takes the form

$$
x_{i}=\frac{q_{i}(\mathbf{u})}{r(\mathbf{u})} \quad(i=1, \ldots, n+1),
$$

where

$$
q_{i}(\mathbf{u})= \begin{cases}2 u_{i}, & \text { if } 1 \leq i \leq n \\ 1-u_{1}^{2}-\cdots-u_{n}^{2}, & \text { if } i=n+1\end{cases}
$$

and

$$
r(\mathbf{u})=1+u_{1}^{2}+\cdots+u_{n}^{2}
$$

Now note that

$$
p_{1}\left(q_{i} / r, \ldots, q_{n} / r\right)=r^{-k_{1}} \tilde{p}_{1} \quad \text { and } \quad p_{2}\left(q_{1} / r, \ldots, q_{n} / r\right)=r^{-k_{2}} \tilde{p}_{2}
$$

for some polynomials $\tilde{p}_{1}$ and $\tilde{p}_{2}$ in $\mathbb{R}\left[u_{1}, \ldots, u_{n}\right]$ and some non-negative integers $k_{1}$ and $k_{2}$. As $r(\mathbf{u}) \neq 0$ for each $\mathbf{u} \in \mathbb{R}^{n}$, we see that $\tilde{p}_{1}(\mathbf{u}) \tilde{p}_{2}(\mathbf{u})=0$ for each $\mathbf{u} \in \mathbb{R}^{n}$. Since the set of polynomial functions on $\mathbb{R}^{n}$ is isomorphic, 
as a ring, to $\mathbb{R}\left[u_{1}, \ldots, u_{n}\right]$ and since $\mathbb{R}\left[u_{1}, \ldots, u_{n}\right]$ is an integral domain, it follows that either $\tilde{p}_{1}(\mathbf{u})=0$ for each $\mathbf{u} \in \mathbb{R}^{n}$ or $\tilde{p}_{1}(\mathbf{u})=0$ for each $\mathbf{u} \in \mathbb{R}^{n}$. Consequently, either $p_{1}(\mathbf{x})=0$ for each $\mathbf{x} \in \mathbb{S}^{n} \backslash\left\{[0, \ldots, 0,1]^{\top}\right\}$ or $p_{2}(\mathbf{x})=0$ for each $\mathbf{x} \in \mathbb{S}^{n} \backslash\left\{[0, \ldots, 0,1]^{\top}\right\}$. Now, by the continuity of polynomials in the usual Euclidean topology and the fact that the closure of $\mathbb{S}^{n} \backslash\left\{[0, \ldots, 0,1]^{\top}\right\}$ in the usual topology is equal to $\mathbb{S}^{n}$ whenever $n \geq 1$, a polynomial which vanishes on $\mathbb{S}^{n} \backslash\left\{[0, \ldots, 0,1]^{\top}\right\}$ vanishes on the whole of $\mathbb{S}^{n}$. This implies that either $p_{1}$ or $p_{2}$ vanishes identically on $\mathbb{S}^{n}$. The proof is complete.

\section{References}

[1] Benedetti, R., Risler, J.J.: Real Algebraic and Semi-Algebraic Sets. Hermann, Paris (1990)

[2] Bochnak, J., Coste, M., Roy, M.F.: Real Algebraic Geometry. Springer, Berlin (1998)

[3] Chen, P., Suter, D.: Rank constraints for homographies over two views: revisiting the rank four constraint. Int. J. Computer Vision 81(2), 205-225 (2009)

[4] Chojnacki, W., Szpak, Z.L., Brooks, M.J., van den Hengel, A.: Multiple homography estimation with full consistency constraints. In: Proc. Digital Image Computing: Techniques and Applications Conf., pp. 480-485 (2010)

[5] Eriksson, A., van den Hengel, A.: Optimization on the manifold of multiple homographies. In: Proc. IEEE 12th Int. Conf. Computer Vision Workshops, pp. 242-249 (2009)

[6] Guillemin, V., Pollack, A.: Differential Topology. Prentice-Hall Inc., Englewood Cliffs, N.J. (1974)

[7] Harris, J.: Algebraic Geometry. Springer, New York (1995)

[8] Hartley, R.I., Zisserman, A.: Multiple View Geometry in Computer Vision, 2nd edn. Cambridge University Press, Cambridge (2004)

[9] Lütkepol, H.: Handbook of Matrices. John Wiley \& Sons, Chichester (1996)

[10] Shashua, A., Avidan, S.: The rank 4 constraint in multiple ( $\geq 3)$ view geometry. In: Proc. 4th European Conf. Computer Vision, Lecture Notes in Computer Science, vol. 1065, pp. 196-206 (1996)

[11] Tu, L.W.: An Introduction to Manifolds. Springer, New York (2008)

[12] Zelnik-Manor, L., Irani, M.: Multiview constraints on homographies. IEEE Trans. Pattern Anal. Mach. Intell. 24(2), 214-223 (2002)

Wojciech Chojnacki

School of Computer Science, The University of Adelaide, SA 5005, Australia and

Wydział Matematyczno-Przyrodniczy, Szkoła Nauk Ścisłych, Uniwersytet Kardynała Stefana Wyszyńskiego, Dewajtis 5, 01-815 Warszawa, Poland

e-mail: wojciech.chojnacki@adelaide.edu.au

Anton van den Hengel

School of Computer Science, The University of Adelaide, SA 5005, Australia e-mail: anton.vandenhengel@adelaide.edu.au 